\title{
Exploring Predictability of SAT/SMT Solvers
}

\author{
Robert Brummayer \\ Johannes Kepler University \\ robert.brummayer@jku.at \\ The University of Iowa \\ Iowa City. Iowa, USA \\ duckki-oe@uiowa.edu \\ and Aaron Stump \\ The University of Iowa \\ astump@acm.org
}

\begin{abstract}
This paper seeks to explore the predictability of SAT and SMT solvers in response to different kinds of changes to benchmarks. We consider both semantics-preserving and possibly semantics-modifying transformations, and provide preliminary data about solver predictability. We also propose carrying learned theory lemmas over from an original run to runs on similar benchmarks, and show the benefits of this idea as a heuristic for improving predictability of SMT solvers.
\end{abstract}

\section{Motivation}

SAT and SMT (Satisfiability Modulo Theories) solvers have enjoyed tremendous performance improvements in the past ten years, increasing the automated-reasoning power available for applications like algorithmic verification, combinatorial design, planning, and others (e.g., 7 [5, 4]). Most work in the field has focused just on performance-oriented quality metrics for solvers. For example, the basic measure used in both the most recent (at the time of writing) SAT Competition and SMT Competition was simply the pair of the number of benchmarks solved and running time to solve them, compared in the natural lexicographic order (for the competitions mentioned, see, e.g., 1, 3]). While the SAT competition has also experimented recently with more complex measures, they are also centered on performance.

In this paper, we propose another property to consider when evaluating solvers, namely predictability. While users certainly require and benefit from improvements to raw performance, anecdotal evidence from end users of solvers suggests that in some cases unpredictability is at least as significant a concern. For example, Steve Miller, Principal Software Engineer in the Advanced Technology Center of Rockwell Collins, reported in his keynote presentation at Midwest Verification Day 2009 that unpredictability is a significant issue for his team in incorporating SAT/SMT solvers into their verification workflow. Unpredictability is a problem because a small change to a model can lead to an enormous change in the amount of time to solve the resulting verification condition. If the amount of time is enormously longer, the verification may become infeasible or unacceptably delayed. If it is enormously shorter, engineers may doubt the result, questioning if an error elsewhere in the workflow has led to such different system behavior. It may improve the usability of such solvers to sacrifice a modest amount of performance for improved predictability.

In this paper we provide a preliminary study of predictability of SAT (Section 2) and SMT (Section 3) solvers under small mutations of standard benchmarks. We use the standard deviation of solver times on a collection of mutants as a measure of variability. In the case of SMT solvers, we also propose and study a technique for heuristically improving predictability, by carrying over a selection of learned theory lemmas from the original run of the solver to the runs on the mutants. 


\section{Experiments with SAT Benchmarks}

We survey how small changes to benchmarks affect the performance of SAT solvers. In particular, we evaluate the effects of semantics-preserving changes such as variable renaming and literal/clause reordering. Moreover, changes that may change the satisfiability status, e.g. adding resp. dropping arbitrary literals, are evaluated. The goal is to quantify the variability of solving times and to identify the effects of different types of variations.

\subsection{Methods}

From the SAT competition 2009, 5 solvers and 13 benchmarks were chosen. The solvers are some of the highly ranked ones in each category, and the benchmarks are of easy to medium difficulty that could be solved in about 300 seconds from the industrial category of the competition. Those solvers and benchmarks are listed below with solving times:

\begin{tabular}{|l|l|l|l|l|l|}
\hline Benchmark & CirCUs & lysat & MiniSat & mxc-sat09 & precosat \\
\hline ACG-10-5p0 & 44.72 & 39.99 & 21.52 & 47.26 & 35.59 \\
AProVE09-07 & 7.08 & 12.25 & 4.72 & 7.06 & 2.89 \\
AProVE09-17 & 13.44 & 9.62 & 7.33 & 6.75 & 4.95 \\
AProVE09-20 & 292.43 & 45.25 & 16.64 & 28.08 & 35.14 \\
countbitsrotate016 & 27.94 & 61.81 & 24.2 & 11.7 & 14.5 \\
gss-14-s100 & 65.2 & 22.79 & 18.37 & 13.54 & 47.97 \\
gus-md5-04 & 4.92 & 5.62 & 2.17 & 16.91 & 5.3 \\
icbrt1_32 & 14.59 & 15.29 & 7.72 & 11.61 & 11.34 \\
minand128 & 9.86 & 49.8 & 20.01 & 55.83 & 13.77 \\
minandmaxor032 & 7.73 & 6.23 & 3.65 & 5.51 & 4.92 \\
minxorminand032 & 5.2 & 8.37 & 4.6 & 9.33 & 7.25 \\
minxorminand064 & 106.21 & 157.31 & 106.16 & 327.19 & 151.4 \\
post-c32s-gcdm16-22 & 249.57 & 115.06 & 97.41 & 151.21 & 51.15 \\
\hline
\end{tabular}

Types of variations We made random changes to the original in order to simulate situations in which users query similar, but not identical, formulas repeatedly. Seven types of variations were performed and summarized in Figure 1. The first five variations preserve the semantics of the original formula and do not change the satisfiability status of the formula. In contrast, the variations $n l c x$ and nlca may change the satisfiability status. For the variation nlcx, $0.01 \%$ of all non-unary clauses are considered small and an arbitrary literal (of the existing variables) replaces one random literal of each affected clause. The variation nlca performs even more changes. In particular, the same number of binary and ternary clauses are changed with probability $0.01 \%$. One literal is added to each of those binary clauses and one literal is dropped from each of those ternary clauses. Note that we tried to avoid some of the possible unrealistic changes. Unary clauses, the literal-clause ratio, and the lengths of clauses are left unchanged in order to keep the inherent difficulty level of the formula.

Measure of Predictability For each type of variation and each benchmark, a random sample of 50 variations was generated and the solving times were recorded. Each solver has its own performance distribution over the same sample. For predictability, we only care about the spread of distribution or the variability of data. If the distribution of a solver is "narrower", we can say the solver is more predictable. To statistically quantify variability, we used the standard deviation of solving times. Obviously, a "small" standard deviation indicates high predictability. 


\begin{tabular}{|c|l|}
\hline Type & Description \\
\hline$l$ & literals in each clause are reordered \\
$c$ & clauses of the formula are reordered \\
$n$ & variable names are changed \\
$l c$ & a combination of $l$ and $c$ variations \\
$n l c$ & a combination of $n, l$ and $c$ variations \\
$n l c x$ & in addition to $n l c$, one literal of non-unary clause is changed $(0.01 \%$ of chance $)$ \\
$n l c a$ & in addition to $n l c$, one literal is dropped from or added to clause $(0.01 \%$ of chance $)$ \\
\hline
\end{tabular}

Figure 1: Types of variations

\section{$2.2 \quad$ Empirical Results}

Figure 2 summarizes the variabilities of solving times induced by the different types of variation. For each variation and each solver, the standard deviations of solving times for all benchmarks were collected. Each bar in the graphs summarizes the distribution of the 13 standard deviations for a given solver and a given variation. The gray box of each bar represents the range of the middle half values, which are considered typical values. The line in the middle of box marks the median value and the "+" sign marks the average. The whiskers sticking out of box extend to adjacent values that are not farther away from the edge of the box than 1.5 times the height of the box. Small squares are values farther out than the adjacent values and considered outliers.

The result for the variation $l$ shows that all solvers have small variability compared to those under other types of variations. Note that the scale of the graph is smaller than the others. Interestingly, reordering literals affects the predictability of precosat more than other solvers. This could be easily avoided by sorting the literals inside the SAT solver. The outstandingly high value of CirCUs is for AProVE09-20. Notably, the solver showed less predictability over all variations of that particular benchmark. The results for the other semantics-preserving variations are almost the same. All solvers showed very similar behavior in general, except a few outliers. The other notable outlier, which belongs to mxc-sat09, is minxorminand064. Our experimental results suggest that any single type of variation, except for $l$, is sufficient to shuffle the performance of solvers without changing semantics. More experiments are necessary to generalize this observation to other benchmarks.

The nlcx variation changed the variabilities for some benchmarks so that more outliers appear in the graphs. Interestingly, the highest outliers are all for post-c32s-gcdm16-22. However, the majority of benchmarks did not change the variability of solving times compared to the result for the variation type $n l c$. Interestingly, the nlca variation uniformly made the formula easier to solve and the solving times less variable across all solvers. At the same time, relative variability among solvers did not change much. Therefore, the graph looks similar to that of $n l c x$ even if the scale of graph is different. Considering this variation, the highest outliers are all for AProVE09-20.

\section{Experiments with SMT Benchmarks and Theory Lem- mas}

In this section, we explore mutation of SMT benchmarks, taken from the SMT-LIB library [2]. The following mutations are applied below with equal probability: (1) change the value of a real 

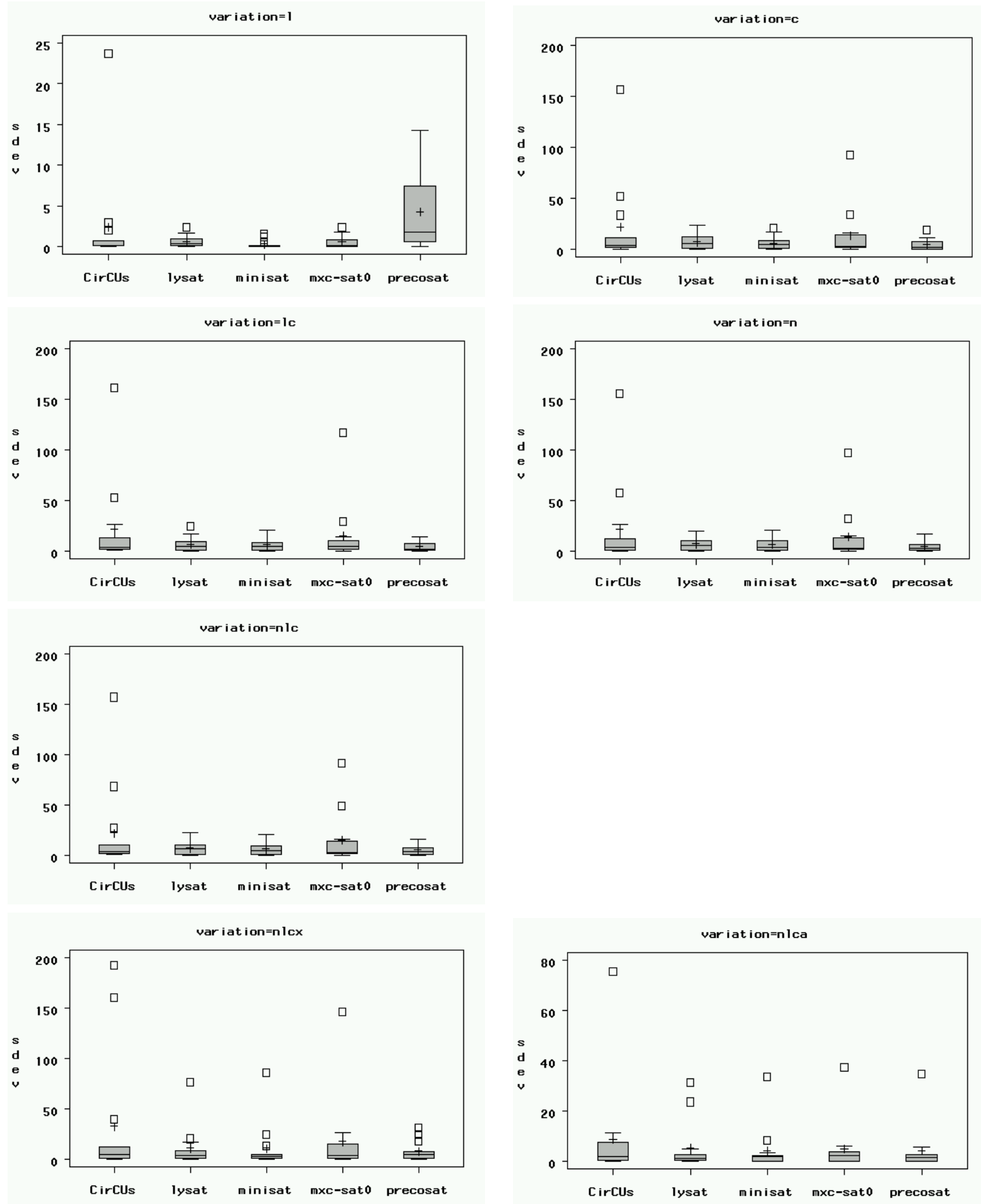

Figure 2: The variabilities of solving times by different types of variation 
or integer constant; (2) swap operands of a predicate or function symbol, or logical connective; (3) change a predicate or function symbol, or logical connective, to one of the same type; (4) insert a logical negation; and (5) perform a local rewriting step to change a formula or a term to an equivalent one. Only (5) is semantics-preserving in general.

The goal of this experiment is to assess the impact of inserting theory lemmas dumped from a run of the solver on the original benchmark, on mutations of that benchmark. The rationale for inserting learned theory lemmas as possibly improving performance and/or predictability is that learned theory lemmas represent information the solver found useful when solving the original benchmark. The idea is that this information may also prove useful when solving a similar benchmark. It is also possible that the learned lemmas will mitigate the effect of the mutation.

Previous work by Whittemore et al. on reusing derived lemmas for SAT for a similar scenario (solving a sequence of related instances) requires tracking which lemmas are invalidated by changes in the formula 6 . In contrast, theory lemmas are, by definition, formulas which are valid in the solver's (possibly combined) background theory, without any other assumptions. Thus, it is always semantics-preserving to add a theory lemma, as it is always true modulo the theory, regardless of the rest of the benchmark formula. So no tracking is needed for theory lemmas (but would be needed for lemmas that follow from the input formula).

We modified the two open-source SMT solvers CVC3 and OPENSMT to dump learned theory lemmas, with the helpful advice of the authors of those tools. We then perform the following test for selected benchmarks (discussed below), using a timeout of 1 minute and a memory limit of 1GB:

1. Run the solver on the original benchmark. If this times out, abort the rest of the test.

2. Run the lemma-dumping modified version of the solver to generate theory lemmas (all other runs use the unmodified solver).

3. Insert theory lemmas into the benchmark as additional assumptions to obtain a modified benchmark. Run the solver on this modified benchmark.

4. Generate 11 mutants from the original benchmark, using the above mutations. For these experiments, we allowed 4 changes to each benchmark. For all divisions exception QF_RDL, we used 2 changes to formula structure, and 2 changes to term structure. For QF_RDL, changes to the term structure tend to take the benchmark out of the syntactic class for difference logic, so for QF_RDL we made 4 changes to the formula structure only.

5. Run the solver on each generated mutant.

6. Insert the lemmas dumped for the original benchmark into each mutant, and run the solver on each of the resulting benchmarks.

Dumping theory lemmas. As mentioned, we modified CVC3 and opensmt to dump learned theory lemmas, following helpful advice from the authors of those tools. Early experiments showed that inserting all learned theory lemmas into benchmarks tends to overwhelm the solver. So we just dump $10 \%$ of the learned theory lemmas. For CVC3, we dumped every 10th learned lemma. For OPEnSMT, we dumped the $10 \%$ of learned theory lemmas with at most 2 literals which had the highest activity (as measured by opensmt's internal measure of activity). Certainly one could be interested to compare alternative methods for selecting theory lemmas to dump. However, this is scheduled as future work. OPENSMT does not normally learn theory 


\begin{tabular}{|l|lll|ll|ll|l|l|}
\hline name & orig & orig+lem & $\mathrm{L}$ & $\tilde{\mathrm{m}}$ & $\sigma_{m}$ & $\tilde{\mathrm{l}}$ & $\sigma_{l}$ & $m / l$ & $\sigma_{m} / \sigma_{l}$ \\
\hline LamportBakery14 & 3.26 & 6.1 & 44 & 2.49 & 0.4 & 2.46 & 1.01 & & \\
LamportBakery15 & 2.19 & 2.22 & 58 & 1.97 & 0.29 & 1.93 & 0.27 & 1.02 & 1.05 \\
OOO5 & 3.16 & 2.56 & 62 & 2.66 & 0.37 & 2.55 & 0.16 & 1.05 & 2.23 \\
sorted_list_insert_noalloc3 & 4.86 & 4.52 & 61 & 4.13 & 0.37 & 4.34 & 0.36 & & 1.03 \\
vhard8 & 2.01 & 7.75 & 306 & 0.07 & 0.01 & 0.66 & 0.04 & & \\
OOO8 & 3.71 & 8.57 & 40 & 3.51 & 0.51 & 3.62 & 2.31 & & \\
ibm_cache_full_q_unbounded12 & 4.19 & 6.07 & 49 & 4.2 & 0.24 & 5.47 & 0.96 & & \\
sorted_list_insert_noalloc5 & 4.74 & 4.48 & 93 & 3.94 & 0.44 & 4.36 & 0.33 & & 1.32 \\
OOO6 & 3.22 & 6.43 & 40 & 2.78 & 0.41 & 3.99 & 1.44 & & \\
sorted_list_insert_noalloc6 & 7.03 & 4.42 & 239 & 3.79 & 1.4 & 4.26 & 0.44 & 1.15 & 3.15 \\
vhard5 & 0.49 & 1.08 & 85 & 0.04 & 0.01 & 0.15 & 0.03 & & \\
ibm_cache_full_q_unbounded15 & 2.85 & 2.36 & 81 & 2.87 & 0.92 & 2.24 & 0.82 & 1.2 & 1.12 \\
ibm_cache_full_q_unbounded14 & 4.04 & 4.16 & 35 & 4.05 & 0.75 & 4.13 & 0.36 & & 2.06 \\
vhard6 & 0.85 & 2.16 & 138 & 0.04 & 0.01 & 0.27 & 0.59 & & \\
ibm_cache_full_q_unbounded17 & 7.78 & 19.83 & 114 & 4.07 & 2.04 & 2.27 & 5.63 & & \\
ibm_cache_full_q_unbounded16 & 4. & 4.04 & 35 & 4.01 & 0.74 & 4.04 & 0.17 & 1.06 & 4.3 \\
vhard16 & 19.3 & 120. & 2235 & 0.16 & 0. & 7.43 & 0.01 & & \\
vhard9 & 2.77 & 15.39 & 427 & 0.08 & 0.01 & 0.95 & 0.03 & & \\
vhard18 & 29.23 & 120. & 3151 & 0.19 & 0. & 13.08 & 0.03 & & \\
vhard11 & 5.03 & 31.53 & 760 & 0.12 & 0.85 & 1.79 & $33.98(1)$ & & \\
\hline
\end{tabular}

Figure 3: Results for CVC3: QF_UFIDL

lemmas outright (but rather, lemmas derived from theory lemmas by conflict analysis). We configured OPENSMT to learn theory lemmas of length at most 2, and kept that configuration for all runs of OPENSMT reported below.

Benchmark selection. The tests below were performed on a selection of benchmarks used in SMT-COMP 2009 (see, e.g., 1] and earlier papers for more on SMT-COMP). The selection process used was the following. We are looking at several mature example divisions: QF_UFIDL, QF_AUFLIA, QF_LIA, QF_LRA, and QF_RDL. CVC3 competed in all those divisions, while of these, opensmt competed just in QF_LRA and QF_RDL. For each solver and division in which it competed, we consider those benchmarks which it could solve in time between 1 second and 1 minute. A further issue we dealt with is that both SMT solvers sometimes introduce new symbols which make their way into theory lemmas. This is problematic, because the meanings and types of those symbols are not determined by the original benchmark. In the end, the approach we adopted was to try to translate away ites (term-level if-then-else expressions) from benchmarks, since these seem to be the biggest (but not only) source of new symbols showing up in theory lemmas. CVC3 recently added a command-line option +liftITE that can be used to do this. In some cases, lifting ites results in an explosion in the size of the formula, crashing the translating invocation of CVC3. In such cases, we excluded the benchmarks from our sample. The test machine for the experiments was a lightly-loaded Intel Core Dual CPU at $1.2 \mathrm{GHz}$, with $1.5 \mathrm{~GB}$ physical memory.

\subsection{Empirical Results}

Figures 3 through 10 summarize the results of these experiments (Figures 6 through 10 are relegated to the appendix for space reasons). Each figure corresponds to a single division and a single solver, except that for typographic reasons, the results for OPENSMT on QF_RDL are split over Figures 9 and 10. Each row in the table corresponds to a test on a single benchmark, as 


\begin{tabular}{|l|lll|ll|ll|l|l|}
\hline name & orig & orig+lem & $\mathrm{L}$ & $\tilde{\mathrm{m}}$ & $\sigma_{m}$ & $\tilde{\mathrm{l}}$ & $\sigma_{l}$ & $m / l$ & $\sigma_{m} / \sigma_{l}$ \\
\hline ParallelPrefixSum_live_blmc002 & 2.25 & 2.21 & 2 & 2.27 & 0.04 & 2.26 & 0.04 & & 1.01 \\
ParallelPrefixSum_safe_blmc008 & 9.57 & 9.49 & 6 & 9.55 & 0.59 & 9.62 & 0.56 & & 1.04 \\
FISCHER11-7-fair & 99.89 & 15.67 & 180 & 92.08 & $47.59(1)$ & 17.66 & 4.59 & & \\
FISCHER6-10-fair & 69.24 & 41.13 & 293 & 56.03 & 29.68 & 52.89 & 23.86 & 1.32 & 1.24 \\
\hline
\end{tabular}

Figure 4: Results for CVC3: QF_LIA

\begin{tabular}{|l|lll|ll|ll|l|l|}
\hline name & orig & orig+lem & $\mathrm{L}$ & $\tilde{\mathrm{m}}$ & $\sigma_{m}$ & $\tilde{\mathrm{l}}$ & $\sigma_{l}$ & $m / l$ & $\sigma_{m} / \sigma_{l}$ \\
\hline storeinv_t1_pp_nf_ai_00009_001 & 47.6 & 16.19 & 11176 & 0.13 & 23.18 & 0.85 & 7.74 & 2.68 & 2.99 \\
swap_t1_pp_nf_ai_00009_007 & 4.32 & 8.31 & 100 & 4.34 & 1.91 & 7.85 & 3.53 & & \\
pointer-safe-10 & 9.54 & 2.87 & 102 & 5.2 & 3.94 & 1.72 & 3.65 & 1.76 & 1.08 \\
pointer-invalid-20 & 117.29 & 38.92 & 721 & 68.36 & $47.17(4)$ & 31.46 & $32.88(1)$ & & \\
pointer-invalid-10 & 10.72 & 1.17 & 126 & 4.65 & 4.7 & 1.73 & 4.1 & 1.97 & 1.14 \\
pointer-safe-15 & 41.1 & 28.25 & 277 & 20.78 & 27.07 & 7.44 & 18.62 & 1.67 & 1.45 \\
qlock-bug-5 & 19.48 & 5.7 & 312 & 17.86 & $37.84(1)$ & 5.72 & $34.03(1)$ & & \\
swap_t1_pp_nf_ai_00009_002 & 23.6 & 18.33 & 103 & 23.61 & 10.84 & 17.06 & 7.84 & 1.37 & 1.38 \\
pointer-invalid-15 & 42.07 & 10.88 & 328 & 21.64 & 27.75 & 8.93 & 28.76 & 1.7 & \\
qlock.base.5 & 14.68 & 12.45 & 215 & 12.77 & $33.94(1)$ & 1.94 & $33.49(1)$ & & \\
qlock-mutex-5 & 14.81 & 7.31 & 225 & 12.73 & $38.35(1)$ & 1.85 & $33.6(1)$ & & \\
\hline
\end{tabular}

Figure 5: Results for CVC3: QF_AUFLIA

described above. All times are given in seconds. The headings in the figure are as follows:

- name: the of the benchmark.

- orig: the time for the (unmodified) solver to solve the benchmark.

- orig+lem: the time for the solver to solve the modified version of the original benchmark, with theory lemmas inserted.

- L: the number of lemmas produced by the run of the lemma-generating version of the solver.

- $\tilde{\mathrm{m}}$ : the median of the times to solve the 11 generated mutants.

- $\sigma_{m}$ : the standard deviation of those times.

- $\tilde{\mathrm{l}}$ : the median of the times to solve the 11 mutants with the same set of lemmas as above inserted into each mutant.

- $\sigma_{l}$ : the standard deviation of those times.

- $m / l$ : the ratio of total time to solve the 11 mutants to the total time to solve the 11 mutants with lemmas inserted. For readability, we only list this number (and similarly for the next column, for $\sigma_{m} / \sigma_{l}$ ) for rows where we have no missing data, and if it is greater than 1 . This quantity represents the speedup using lemmas, and so we view this as a relative performance metric.

- $\sigma_{m} / \sigma_{l}$ : the ratio of the above-defined standard deviations. We view this as a relative predictability metric. 
Missing data. We lose data in this experiment for timeouts and memory outs, indicated in parentheses with the standard deviation; and occasionally where mutation takes a benchmark out of the syntactic class for the division, indicated in square brackets with the standard deviation. The latter problem just occurs for OPENSMT on QF_LRA, where the mutation occasionally creates divisions by zero.

Some observations about the data in the figures are warranted. First, it is not generally the case that either $m / l$ or $\sigma_{m} / \sigma_{l}$ is improved by inserting theory lemmas. The ratio $\sigma_{m} / \sigma_{l}$ (computed only for tests with no censored data) is greater than 1 for $36 \%$ of the 68 total benchmarks (including tests with censored data). Similarly, the ratio $m / l$ is greater than 1 for $35 \%$. We can observe, however, that in some cases, one or the other, or both, measures are improved. For example, in Figure 3, the 0005 benchmark shows a modest improvement in performance but a significant $(>2 \mathrm{x})$ improvement in predictability. In some cases, such as sorted_list_insert_noalloc5, predictability improves even with an overall decline in performance. We can also see that inserting theory lemmas back into the original benchmark, while sometimes resulting in a significant slowdown (e.g., around 2x for LamportBakery14 of Figure 3) can sometimes lead to big performance improvements: consider, for example, the results for FISCHER11-7-fair (Figure 4), where inserting theory lemmas leads to roughly a $6 \mathrm{x}$ speedup over the original benchmark; or for pointer-invalid-15 (Figure 5), with around a $4 \mathrm{x}$ speedup.

So insertion of theory lemmas, while not generally helpful for performance or predictability, may have value as a heuristic for improving both. In our target use-model, a team making repeated calls to a solver can simply turn on "retain-lemmas" mode, and see if it improves performance or, over several runs, predictability. If not, the heuristic can be turned off. But if so, it may improve the end-user's experience for subsequent runs of the solver.

\section{Conclusion and Future Work}

We have considered preliminary data studying how various mutations to benchmarks affect the performance and reveal the predictability of SAT and SMT solvers, on collections of standard benchmarks. We have also considered one heuristic for improving predictability of SMT solvers, namely retaining learned theory lemmas from an original run to subsequent runs on similar benchmarks. This corresponds to a scenario where successive changes made by an end-user cause modest changes to the benchmark formula.

For future work, a more informed model for mutation is required, to ensure that the proposed methods apply in real-world situations. For such a model, it would be very useful to have or to be able to generate a sequence of formulas from successive small modifications to a verification model or other application-specific structure. This will enable more accurate further studies of methods to measure and improve solver predictability. One possibility might be to consider benchmarks from the same benchmark family (for both the SAT and SMT experiments), since these should exhibit some similarities. For the SMT experiments, it would be interesting to test whether or not adding theory lemmas learned from a run of the SMT solver on one benchmark can improve predictability for other benchmarks from the same family. Similarly, it would be interesting to do more thorough exploration of which lemmas to carry over from one run to the next, and whether lemmas learned by one solver can improve predictability for another.

Acknowledgments. Thanks to Clark Barrett and Roberto Bruttomesso for help modifying CVC3 and opensmt, respectively. Thanks also to Alberto Segre for consultation on statistics, and to Cesare Tinelli for discussion of the idea of considering predictability for SMT solvers. 


\section{References}

[1] Clark Barrett, Morgan Deters, Albert Oliveras, and Aaron Stump. Design and results of the $3^{\text {rd }}$ annual satisfiability modulo theories competition (SMT-COMP 2007). International Journal on Artificial Intelligence Tools (IJAIT), 17(4):569-606, August 2008.

[2] Clark Barrett, Silvio Ranise, Aaron Stump, and Cesare Tinelli. The Satisfiability Modulo Theories Library (SMT-LIB). www. SMT-LIB .org, 2008.

[3] D. Berre and L. Simon. 55 Solvers in Vancouver: The SAT 2004 competition. In H. Hoos and D. Mitchell, editors, Proceedings of the International Conference on Theory and Applications of Satisfiability Testing (SAT), 2004.

[4] E. Giunchiglia and M. Maratea. Planning as Satisfiability with Preferences. In Proceedings of the Twenty-Second AAAI Conference on Artificial Intelligence., pages 987-992. AAAI Press, 2007.

[5] S. Lahiri, S. Qadeer, and Z. Rakamaric. Static and Precise Detection of Concurrency Errors in Systems Code Using SMT Solvers. In A. Bouajjani and O. Maler, editors, Computer Aided Verification, 21st International Conference, CAV 2009. Proceedings, pages 509-524, 2009.

[6] J. Whittemore, J. Kim, and K. Sakallah. Satire: A new incremental satisfiability engine. In Design Automation Conference, 2001. Proceedings, pages 542 - 545, 2001.

[7] H. Zhang. Combinatorial designs by SAT solvers. In A. Biere, M. Heule, H. van Maaren, and T. Walsh, editors, Handbook of Satisfiability, volume 185 of Frontiers in Artificial Intelligence and Applications, chapter 17, pages 533-568. IOS Press, 2009.

\section{A More Results from SMT Experiments}




\begin{tabular}{|c|c|c|c|c|c|c|c|c|c|}
\hline name & orig & orig+lem & $\mathrm{L}$ & $\tilde{\mathrm{m}}$ & $\sigma_{m}$ & I & $\sigma_{l}$ & $m / l$ & $\sigma_{m} / \sigma_{l}$ \\
\hline sc-35.induction3 & 5.02 & 120 & 94 & 1.45 & 2.72 & 1.48 & $57.06(4)$ & & \\
\hline sc-15.induction & 1.49 & 0.94 & 16 & 0.32 & 0.54 & 0.33 & 0.36 & 1.24 & 1.5 \\
\hline uart-7.induction & 2.08 & 1.78 & 78 & 0.67 & 0.67 & 0.64 & 0.66 & 1.05 & \\
\hline sc-12.induction & 1.17 & 0.67 & 14 & 0.26 & 0.4 & 0.27 & 0.24 & 1.23 & 1.65 \\
\hline p2-zenonumeric_s6 & 2.79 & 7.77 & 27 & 1.78 & 0.4 & 3.23 & 1.54 & & \\
\hline uart-6.induction & 1.62 & 1.07 & 69 & 0.49 & 0.51 & 0.53 & 0.32 & 1.07 & 1.56 \\
\hline sc-14.induction & 1.33 & 0.86 & 16 & 0.32 & 0.5 & 0.3 & 0.32 & 1.29 & 1.56 \\
\hline reint_to_least.base & 7.06 & 7.2 & 30 & 0.21 & 0.79 & 0.23 & $34.31(1)$ & & \\
\hline pursuit-safety-7 & 5.38 & 1.03 & 50 & 1.65 & 8.9 & 0.58 & 0.46 & 6.22 & 19.28 \\
\hline sc-18.induction & 1.91 & 1.18 & 19 & 0.4 & 0.76 & 0.41 & 0.46 & 1.31 & 1.64 \\
\hline sc-10.induction & 0.79 & 0.54 & 12 & 0.22 & 0.26 & 0.24 & 0.19 & 1.14 & 1.35 \\
\hline uart-9.induction & 2.98 & 3.32 & 100 & 0.92 & 1.04 & 1.09 & 1.27 & & \\
\hline p-0-bucket_s7 & 5.3 & 4.6 & 58 & 5.33 & 0.04 & 4.74 & 0.39 & 1.1 & \\
\hline gasburner-prop3-16 & 0.75 & 0.77 & 20 & 0.53 & 19.63 & 0.63 & 12.71 & 1.46 & 1.54 \\
\hline gasburner-prop3-17 & 0.79 & 1.07 & 21 & 0.66 & $34.16(1)$ & 0.72 & $36.55(1)$ & & \\
\hline clocksynchro_3clocks.main_invar.base & 1.05 & 1.09 & 18 & 0.23 & 0.07 & 0.27 & 0.24 & & \\
\hline p-driverlogNumeric_s7 & 61.48 & 120. & 21 & 3.59 & 4.1 & 6.59 & $32.75(1)$ & & \\
\hline tgc_io-safe-13 & 11.41 & 6.6 & 70 & 2.03 & 2.41 & 2.83 & 3.26 & & \\
\hline sc-24.induction & 3.37 & 1.93 & 25 & 0.58 & 1.48 & 0.7 & 0.75 & 1.42 & 1.95 \\
\hline clocksynchro_9clocks.main_invar.base & 7.78 & 7.72 & 51 & 0.78 & 0.37 & 0.94 & 2.14 & & \\
\hline p4-zenonumeric_s5 & 59.2 & 90.93 & 68 & 11.49 & 25.67 & 12.33 & 32.11 & & \\
\hline uart-8.base & 17.43 & 15.73 & 279 & 1.61 & 3.54 & 4.44 & 3.57 & & \\
\hline tgc_io-safe-18 & 42. & 13.5 & 120 & 3.55 & 9.65 & 4.8 & $33.05(1)$ & & \\
\hline p6-zenonumeric_s5 & 65.28 & 120. & 99 & 20.06 & $31.61(1)$ & 22.37 & $43.77(3)$ & & \\
\hline sc-8.induction2 & 3.76 & 4.09 & 33 & 0.24 & 1.7 & 0.34 & 1.9 & & \\
\hline simple_startup_3nodes.abstract.induct & 22.71 & 80.12 & 431 & 0.24 & 0.09 & 0.47 & 0.38 & & \\
\hline tgc_io-safe-20 & 68.34 & 5.74 & 144 & 4.85 & 15.38 & 4.96 & $33.37(1)$ & & \\
\hline simple_startup_4nodes.missing.induct & 2.65 & 1.07 & 143 & 0.32 & 0.1 & 0.4 & 0.18 & & \\
\hline uart-24.induction & 18.58 & 60.41 & 435 & 5.16 & 6.02 & 5.22 & 18.92 & & \\
\hline sc-19.induction & 2.12 & 1.26 & 20 & 0.39 & 0.84 & 0.44 & 0.5 & 1.32 & 1.69 \\
\hline sc-21.induction3 & 12.72 & 30.18 & 111 & 0.86 & 32.94 & 0.96 & 14.68 & 1.94 & 2.24 \\
\hline uart-9.base & 39.64 & 25.67 & 457 & 1.46 & 5.02 & 5.28 & 16.33 & & \\
\hline synched.induction & 3.36 & 3.84 & 49 & 0.27 & 2.46 & 0.29 & $34.17(1)$ & & \\
\hline sc-32.induction3 & 4.4 & 120. & 82 & 1.29 & 2.26 & 1.32 & $51.89(3)$ & & \\
\hline uart-20.induction & 13.01 & 29.44 & 318 & 3.56 & 4.18 & 3.74 & 10.54 & & \\
\hline simple_startup_5nodes.m & 7.36 & 3.29 & 333 & 0.45 & 0.17 & 0.61 & 0.47 & & \\
\hline simple_startup_4nodes.abstract.induct & 76.84 & 120. & 1048 & 0.33 & 0.13 & 0.83 & 0.32 & & \\
\hline
\end{tabular}

Figure 6: Results for CVC3: QF_LRA 


\begin{tabular}{|l|lll|ll|ll|l|l|}
\hline name & orig & orig+lem & $\mathrm{L}$ & $\tilde{\mathrm{m}}$ & $\sigma_{m}$ & $\tilde{\mathrm{l}}$ & $\sigma_{l}$ & $m / l$ & $\sigma_{m} / \sigma_{l}$ \\
\hline fischer3-mutex-8 & 9.13 & 14.7 & 197 & 7.44 & 2.54 & 3.47 & 4.18 & 1.28 & \\
orb02_700 & 4.32 & 4.23 & 32 & 4.71 & 0.16 & 4.49 & 0.23 & 1.04 & \\
fischer6-mutex-6 & 7.16 & 14.68 & 169 & 8.17 & 4.3 & 11.2 & 4.85 & & \\
abz6_800 & 4.9 & 5.29 & 36 & 4.02 & 0.21 & 4.36 & 0.23 & & \\
orb07_330 & 5.63 & 6.41 & 42 & 4.7 & 0.42 & 5.36 & 0.42 & & \\
orb05_700 & 74.98 & 57.42 & 115 & 65.43 & $23.57(3)$ & 68.67 & 15.15 & & \\
fischer9-mutex-5 & 9.12 & 15.05 & 126 & 21.87 & 18.73 & 19. & 9. & 2.08 \\
fischer3-mutex-9 & 16.07 & 20.2 & 356 & 7.16 & 5.81 & 6.54 & 6.24 & 1.22 & \\
fischer6-mutex-7 & 23.66 & 32.32 & 406 & 26.93 & 13.73 & 28.67 & 18.02 & & \\
fischer9-mutex-6 & 50.3 & 91.25 & 405 & 50.3 & 14.43 & 66.48 & $34.54(1)$ & & \\
fischer3-mutex-10 & 23.39 & 50.63 & 572 & 19.75 & 9.75 & 5.11 & 16.36 & 1.29 & \\
fischer6-mutex-8 & 103.89 & 120. & 1209 & 42.34 & $35.89(1)$ & 45.87 & $38.81(2)$ & & \\
abz5_1400 & 27.59 & 120. & 64 & 23.5 & 2.16 & 120. & $0 .(11)$ & & \\
orb09_1100 & 59.45 & 120. & 84 & 27.74 & 17.18 & 120. & $0 .(11)$ & \\
abz5_1000 & 12.53 & 21.89 & 59 & 39.31 & 10.57 & 29.32 & 6.32 & 1.2 & 1.67 \\
fischer3-mutex-12 & 93.66 & 120. & 1319 & 58.14 & 28.9 & 10.82 & $43.58(1)$ & & \\
fischer3-mutex-13 & 118.06 & 120. & 1734 & 100.14 & $44.77(3)$ & 16.17 & $48.22(3)$ & & \\
fischer3-mutex-11 & 40.47 & 119.12 & 902 & 17.9 & 19.61 & 9.07 & $35.27(1)$ & & \\
orb04_850 & 25.93 & 42.75 & 53 & 19.98 & 3.02 & 42.59 & 10.21 & & \\
orb04_1200 & 29.05 & 120. & 63 & 26.81 & 2.21 & 120. & $0 .(11)$ & & \\
orb06_1200 & 46.71 & 120. & 78 & 82.05 & 25.62 & 120. & $4.77(10)$ & & \\
orb05_1000 & 37.57 & 120. & 62 & 60.41 & 13.01 & 120. & $27.95(7)$ & & \\
orb10_1100 & 37.18 & 120. & 68 & 36.09 & 0.61 & 85.53 & $18.52(3)$ & & \\
\hline
\end{tabular}

Figure 7: Results for CVC3: QF_RDL 


\begin{tabular}{|c|c|c|c|c|c|c|c|c|c|}
\hline name & orig & orig+lem & $\mathrm{L}$ & $\tilde{\mathrm{m}}$ & $\sigma_{m}$ & 1 & $\sigma_{l}$ & $m / l$ & $\sigma_{m} / \sigma_{l}$ \\
\hline sc-10.induction & 6.07 & 7.55 & 15 & 0.2 & 2.89 & 0.2 & 3.35 & & \\
\hline pursuit-safety-10 & 1.27 & 1.36 & 18 & 0.54 & 1.33 & 0.59 & 1.06 & 1.1 & 1.25 \\
\hline p5-zenonumeric_s5 & 3.29 & 3.66 & 37 & 3.26 & 0.06 & 3.66 & 0.12 & & \\
\hline pursuit-safety-11 & 1.42 & 1.65 & 17 & 0.66 & 2.87 & 0.74 & 1.72 & 1.35 & 1.66 \\
\hline uart-8.base & 2.93 & 4.12 & 16 & 3.32 & 1.98 & 2.83 & 1.88 & 1.04 & 1.05 \\
\hline sc-11.induction & 12.33 & 8.77 & 17 & 0.22 & 4.9 & 0.22 & 5.02 & & \\
\hline p-0-bucket_s10 & 3.74 & 4.42 & 6 & 6.14 & 2.06 & 5.33 & 0.56 & 1.21 & 3.66 \\
\hline p-DepotsNum_s8.msat & 3.54 & 2.57 & 32 & 1.86 & 0.45 & 1.69 & 0.45 & 1.07 & \\
\hline uart-7.induction & 3.38 & 3.06 & 21 & 0.15 & 1.49 & 0.16 & 1.17 & 1.2 & 1.26 \\
\hline sc-12.induction & 19.71 & 11.25 & 18 & 0.24 & 8.28 & 0.25 & 8.23 & 1.01 & \\
\hline uart-9.base & 6.75 & 8.34 & 21 & 5.01 & 3.05 & 4.24 & 2.84 & 1.1 & 1.07 \\
\hline pursuit-safety-13 & 2.75 & 3.01 & 24 & 1.02 & 5.2 & 0.74 & 4.81 & 1.11 & 1.08 \\
\hline tgc_io-safe-18 & 5.2 & 3.13 & 36 & 3.06 & 1.34 & 3. & 1.18 & & 1.13 \\
\hline clocksynchro_9clocks.main_invar.base & 5.07 & 6.4 & 6 & 0.41 & $3.15[1]$ & 0.53 & $2.79[1]$ & & \\
\hline pursuit-safety-16 & 1.06 & 2.5 & 16 & 1.62 & 12.63 & 1.75 & 20.28 & & \\
\hline tgc_io-safe-20 & 5.7 & 7.15 & 39 & 4.88 & 1.88 & 4.36 & 2.38 & & \\
\hline p-0-bucket_s13 & 8. & 6.24 & 6 & 12.61 & 3.13 & 10.93 & 5.03 & 1.07 & \\
\hline p7-driverlogNumeric_s7 & 5.23 & 12.96 & 45 & 2.19 & 1.51 & 2.39 & 2.27 & & \\
\hline sc-15.induction & 15.12 & 42.9 & 18 & 0.3 & 17.62 & 0.31 & 18.03 & & \\
\hline uart-9.induction & 7.72 & 9.21 & 26 & 0.22 & 3.3 & 0.21 & 2.91 & 1.13 & 1.13 \\
\hline simple_startup_3nodes.abstract.induct & 8.81 & 9.02 & 24 & 0.21 & $0.6[1]$ & 0.19 & $0.52[1]$ & & \\
\hline sc-14.induction & 39.51 & 30.79 & 20 & 0.28 & 11.12 & 0.28 & 13.86 & & \\
\hline sc-12.induction3 & 11.55 & 13.79 & 13 & 0.31 & 6.84 & 0.3 & 4.61 & 1.43 & 1.48 \\
\hline simple_startup_4nodes.missing.induct & 12.17 & 15.59 & 29 & 0.28 & $3.11[1]$ & 0.29 & $2.45[1]$ & & \\
\hline sc-10.base & 14.21 & 13.08 & 15 & 7.03 & 3.95 & 7.96 & 4.67 & & \\
\hline pursuit-safety-15 & 1.67 & 2.25 & 17 & 1.58 & 9.62 & 1.57 & 11.97 & & \\
\hline sc-14.induction3 & 13.74 & 22.94 & 14 & 0.35 & 12.87 & 0.35 & 10.9 & 1.12 & 1.18 \\
\hline sc-18.induction & 110.77 & 116.39 & 25 & 0.38 & 44.05 & 0.37 & 35.59 & 1.22 & 1.23 \\
\hline p-2-bucket_s11 & 26.33 & 24.54 & 8 & 25.81 & 1.52 & 25.28 & 2.01 & & \\
\hline simple_startup_5nodes.missing.induct & 55.16 & 46.07 & 43 & 0.38 & $10.74[1]$ & 0.39 & $11.17[1]$ & & \\
\hline sc-12.base & 32.94 & 33.58 & 19 & 14.3 & 10.19 & 18.71 & 10.88 & & \\
\hline sc-19.induction & 90.19 & 114.56 & 24 & 0.39 & $50.32(1)$ & 0.38 & 49.99(1) & & \\
\hline p7-driverlogNumeric_s8 & 13.03 & 30.67 & 61 & 2.42 & 4.97 & 2.53 & 15.52 & & \\
\hline sc-17.induction2 & 70.35 & 54.6 & 24 & 0.38 & 27.86 & 0.38 & 28.62 & 1.02 & \\
\hline sc-19.induction2 & 60.03 & 120. & 22 & 0.48 & 45.44 & 0.43 & 47.49 & & \\
\hline simple_startup_4nodes.abstract.induct & 50.11 & 44.52 & 38 & 0.28 & $2.83[1]$ & 0.28 & $3.38[1]$ & & \\
\hline opt1217-6 & 45.69 & 42.71 & 313 & 7.43 & 19.52 & 5.15 & 19.72 & 1.02 & \\
\hline uart-13.base & 47.2 & 53.14 & 44 & 17.96 & 12.91 & 17.7 & 10.83 & 1.08 & 1.19 \\
\hline opt1217-11 & 57.04 & 53.3 & 332 & 7.48 & 21.33 & 6.79 & 20.62 & 1.05 & 1.03 \\
\hline sc-14.base & 74.98 & 58.92 & 21 & 29.43 & 20.23 & 36.85 & 19.05 & & 1.06 \\
\hline sc-15.base & 94.18 & 95.45 & 22 & 45.51 & 22.94 & 34.34 & 21.29 & 1.14 & 1.07 \\
\hline
\end{tabular}

Figure 8: Results for opensmt: QF_LRA 


\begin{tabular}{|l|lll|ll|ll|l|l|}
\hline name & orig & orig+lem & $\mathrm{L}$ & $\tilde{\mathrm{m}}$ & $\sigma_{m}$ & $\tilde{\mathrm{l}}$ & $\sigma_{l}$ & $m / l$ & $\sigma_{m} / \sigma_{l}$ \\
\hline orb07_430 & 0.56 & 0.93 & 6 & 0.34 & 0.31 & 0.35 & 0.42 & & \\
orb08_930 & 4.63 & 3.91 & 6 & 1.56 & 1.53 & 1.66 & 5. & & \\
fischer3-mutex-12 & 3.79 & 3.32 & 21 & 0.83 & 0.89 & 1.04 & 0.83 & 1.01 & 1.06 \\
fischer3-mutex-15 & 6.36 & 6.51 & 27 & 1.99 & 1.5 & 1.16 & 1.93 & & \\
orb01_1200 & 0.88 & 0.3 & 6 & 0.35 & 0.23 & 0.31 & 0.05 & 1.42 & 4.51 \\
skdmxa-3x3-6.base & 8.02 & 8.07 & 5 & 8.31 & 2.24 & 8.27 & 2.24 & & \\
fischer3-mutex-14 & 4.49 & 4.02 & 23 & 1.37 & 1.39 & 1.57 & 1.2 & & 1.15 \\
abz6_943 & 3.29 & 3.76 & 6 & 0.38 & 1.17 & 0.4 & 1.3 & & \\
orb10_900 & 2.5 & 3.02 & 6 & 8.93 & 3.05 & 9.81 & 3.25 & & \\
fischer3-mutex-13 & 4.73 & 5.03 & 23 & 1.24 & 1.36 & 0.9 & 0.98 & 1.24 & 1.38 \\
fischer9-mutex-8 & 5.4 & 4.71 & 30 & 1.72 & 1.91 & 1.6 & 1.73 & & 1.1 \\
skdmxa-3x3-7.base & 9.42 & 9.34 & 5 & 10.26 & 2.81 & 10.48 & 2.82 & & \\
fischer6-mutex-9 & 6.6 & 4.27 & 23 & 1.47 & 1.62 & 1.62 & 1.15 & & 1.4 \\
abz7_500 & 5.76 & 5.83 & 6 & 5.7 & 0.95 & 5.79 & 1.22 & & \\
orb09_900 & 6.77 & 6.08 & 7 & 3.36 & 1.7 & 4.2 & 2.55 & & \\
orb10_1000 & 3.62 & 3.09 & 6 & 1.03 & 2.21 & 1.1 & 1.11 & 1.17 & 1.98 \\
skdmxa-3x3-8.base & 12.29 & 11.94 & 5 & 12.95 & 3.8 & 12.85 & 3.75 & & 1.01 \\
orb06_1100 & 2.02 & 3.7 & 6 & 0.81 & 0.85 & 0.54 & 1.14 & & \\
fischer3-mutex-16 & 8.16 & 9.42 & 31 & 2.08 & 2.32 & 1.7 & 2.73 & & \\
orb04_1005 & 27.42 & 6.25 & 9 & 5.26 & 5.07 & 5.47 & $6.22[1]$ & & \\
skdmxa-3x3-9.base & 14.87 & 14.87 & 5 & 16.14 & 4.73 & 16.08 & 4.69 & 1.01 & \\
fischer3-mutex-17 & 10.14 & 7.96 & 29 & 2.21 & 3.02 & 2.36 & 2.34 & 1.11 & 1.28 \\
orb10_944 & 14.39 & 14.64 & 6 & 2.33 & 4.67 & 2.45 & 6.32 & & \\
orb02_888 & 3.56 & 3.33 & 6 & 0.37 & 1.94 & 0.36 & 2.78 & & \\
fischer9-mutex-9 & 10.56 & 18.32 & 36 & 3.22 & 3.92 & 3.89 & 3.32 & & 1.18 \\
fischer3-mutex-18 & 13.05 & 13.37 & 36 & 2.89 & 4.11 & 2.27 & 3.82 & 1.01 & 1.07 \\
skdmxa-3x3-5.induction & 28.12 & 17.71 & 7 & 19.72 & 7.86 & 17.12 & 7.06 & 1.14 & 1.11 \\
skdmxa-3x3-10.base & 19.6 & 19.4 & 5 & 19.71 & 6.05 & 19.96 & 6.05 & & \\
fischer6-mutex-10 & 7.65 & 8.72 & 29 & 2.76 & 2.4 & 2.4 & 2.81 & & \\
\hline
\end{tabular}

Figure 9: Results for opensmt: QF_RDL 


\begin{tabular}{|l|lll|ll|ll|l|l|}
\hline name & orig & orig+lem & $\mathrm{L}$ & $\tilde{\mathrm{m}}$ & $\sigma_{m}$ & $\tilde{1}$ & $\sigma_{l}$ & $m / l$ & $\sigma_{m} / \sigma_{l}$ \\
\hline fischer9-mutex-10 & 22.02 & 22.35 & 45 & 6.04 & 9.93 & 7.94 & 4.99 & 1.4 & 1.98 \\
orb03_1100 & 10.57 & 0.38 & 8 & 0.4 & 1.21 & 0.41 & 0.59 & 1.83 & 2.03 \\
orb07_397 & 10.62 & 17.67 & 6 & 0.66 & 5.9 & 0.78 & 7.12 & & \\
abz5_1200 & 8.91 & 9.01 & 7 & 0.47 & 4.2 & 0.46 & 4.09 & 1.02 & 1.02 \\
orb03_950 & 14.55 & 13.53 & 7 & 11.96 & 1.95 & 11.74 & 3.18 & & \\
fischer3-mutex-19 & 18.64 & 16.42 & 43 & 2.6 & 5.59 & 3.74 & 5.08 & & 1.09 \\
skdmxa-3x3-11.base & 23.17 & 23.28 & 5 & 25.02 & 8.09 & 25.05 & 8. & & 1.01 \\
orb09_934 & 30.93 & 10.48 & 9 & 0.52 & 3.99 & 0.48 & 9.34 & & \\
abz7_800 & 19.33 & 4.34 & 6 & 3.63 & 6.19 & 4.24 & 0.8 & 1.61 & 7.69 \\
orb08_888 & 17.67 & 19. & 8 & 4.52 & 7.19 & 4.66 & 6.74 & 1.07 & 1.06 \\
skdmxa-3x3-12.base & 30. & 30.1 & 5 & 31.34 & 10.19 & 30.93 & 10.08 & 1.01 & 1.01 \\
fischer6-mutex-11 & 12.54 & 26.58 & 35 & 3.3 & 4.36 & 3.69 & 9.3 & & \\
skdmxa-3x3-13.base & 34.69 & 35.08 & 5 & 35.56 & 12.31 & 35.69 & 12.34 & & 1.01 \\
fischer3-mutex-20 & 19.34 & 24.25 & 42 & 2.03 & 6.02 & 3.46 & 5.94 & & \\
orb01_1100 & 21.69 & 18.04 & 8 & 1.19 & 2.23 & 0.88 & 8.21 & & 1.14 \\
fischer6-mutex-12 & 43.47 & 25.85 & 52 & 6.04 & 12.27 & 5.03 & 9.14 & 1.34 \\
orb05_887 & 7.7 & 17.2 & 8 & 0.42 & 7.3 & 0.36 & 5.14 & 1.37 & 1.42 \\
skdmxa-3x3-14.base & 43.51 & 43.06 & 5 & 45.87 & 15.52 & 45.92 & 15.62 & & \\
skdmxa-3x3-5 & 32.3 & 40.95 & 39 & 27.94 & 19.21 & 34.88 & 26.52 & & \\
fischer6-mutex-13 & 30.86 & 52.75 & 54 & 8.69 & 14.24 & 6.94 & 19.28 & & 2.83 \\
abz5_1234 & 30.29 & 17.22 & 9 & 0.38 & 16.33 & 0.4 & 5.76 & 2.68 & \\
skdmxa-3x3-6.induction & 48.16 & 33.19 & 9 & 35.92 & 15.68 & 30.94 & 13.51 & 1.01 & 1.16 \\
skdmxa-3x3-15.base & 56.2 & 55.89 & 5 & 57.54 & 20.43 & 57.21 & 20.48 & & \\
skdmxa-3x3-16.base & 75.21 & 75.66 & 5 & 73.81 & 26.43 & 73.93 & 26.5 & & \\
fischer6-mutex-16 & 108.07 & 120. & 73 & 34.07 & 40.1 & 42.84 & $41.86(1)$ & & \\
fischer9-mutex-11 & 54.97 & 43.05 & 65 & 16.96 & 15.97 & 9.5 & 19.75 & & \\
fischer6-mutex-15 & 83.54 & 78.13 & 70 & 20.37 & 27.43 & 35.25 & 28.53 & & \\
fischer6-mutex-14 & 106.9 & 54.42 & 67 & 17.41 & 17.79 & 14.53 & 19.82 & & \\
\hline
\end{tabular}

Figure 10: Results for opensmt: QF_RDL 